\title{
El soft power \\ en la política exterior de China: consecuencias para América Latina ${ }^{1}$
}

\author{
Isabel Rodríguez Aranda
}

Universidad del Desarrollo, Santiago, Chile. Email: isabelrodriguez@udd.cl

\author{
Diego Leiva Van de Maele
}

Universidad del Desarrollo, Santiago, Chile. Email: investigadorayudante@udd.cl

Resumen: Este artículo analiza la incorporación del soft power como elemento de la política exterior de China para América Latina. Sostenemos que China cuenta con soft power y ha desarrollado una estrategia para proyectarlo en el mundo a partir de su política exterior, basándose en su cultura milenaria, en la cooperación internacional hacia los países en desarrollo y en la promoción de su modelo de desarrollo. Asimismo, la inclusión por parte de China del soft power como elemento de su política exterior en la primera mitad de la década de 2000 tendrá efectos medibles en la intensificación de las relaciones con América Latina en la segunda mitad de esa década. noamericana.

Palabras clave: China, Soft power, política exterior, relación Chino-lati-

\section{The soft power in China's foreign policy: implications for Latin America}

Abstract: This article aims to analyze the incorporation of soft power as an element of China's foreign policy for Latin America. We argue that China has soft power and that has developed a strategy to project in the world from its foreign policy, based on its ancient culture, on international cooperation to developing countries and on the promotion of its development model. Including China's soft power as an element of its foreign policy in the first half of the 2000s, it will have measurable effects on the intensification of its relations with Latin America in the second half of that decade.

Key words: China, Soft power, China's foreign policy, Chino-Latin American relations.

\section{O soft power na política externa da China: implicações para a América Latina}

Resumo: Este artigo analisa a incorporação do soft power, como parte da política externa da China para a América Latina. Argumenta-se que a China tem poder suave e desenvolveu uma estratégia para projetar no mundo a partir de sua política externa, com base em sua cultura milenar na cooperação internacional para países em desenvolvimento e na promoção do seu modelo de desenvolvimento. 
Além disso, a inclusão de poder suave da China como um elemento de sua política externa, na primeira metade da década de 2000 tem efeitos mensuráveis _ sobre a intensificação das relações com a América Latina na segunda metade daquela década.

América Latina

Palavras-chave: China, poder brando, política externa, relações China$* * *$

\section{Introducción}

Este artículo considera la década de 2000 como una nueva etapa en las relaciones sino-latinoamericanas ya que desde entonces se registran relaciones permanentes y de mayor frecuencia en los variados aspectos. En lo económico, el volumen del comercio entre la región y China se incrementó un 1.119,3 \% entre los años 2000 y 2010, y en lo político, existe un interés que responde al anhelo creciente de China de lograr reconocimiento en el sistema internacional y posicionarse en las instituciones multilaterales frente a EE.UU., lo que se ha visto reflejado en una común articulación de una posición de países desarrollados en los procesos e instituciones del sistema internacional, así como en la intensificación de las visitas mutuas de altas autoridades y el establecimiento de acuerdos para establecer mecanismos de diálogo, consulta o cooperación con algunos países y con los bloques subregionales (Rodríguez 2012: 45).

En el contexto de esta nueva etapa es posible argumentar que el interés de China por desarrollar más estrechas relaciones con América Latina (AL) se enmarca en un proceso de transformación de la política exterior de la potencia asiática en cuanto incorpora la variable del soft power en su comprensión de las Relaciones Internacionales, a la vez que despliega su diplomacia pública a regiones de países en desarrollo que necesitan cooperar para insertarse y transformar el sistema internacional a su favor.

El presente artículo, se propone analizar la incorporación del sof power como elemento de la política exterior de China para AL. Sostenemos que China cuenta con soft power y ha desarrollado una estrategia para proyectarlo en el mundo a partir de su política exterior, basándose sobre todo en la riqueza de su cultura milenaria, en sus logros en materia económica -creciendo a tasas de casi un 9\% promedio en los últimos 10 años-, en la cooperación internacional hacia los países en desarrollo (cultural, política, económica y militar), y en la promoción de su modelo de desarrollo. Sin embargo, la ausencia de un sistema político democrático es un factor que disminuye su efecto de atracción, sobre todo en Occidente. Asimismo, China proyecta su soft power en AL, principalmente desde el año 2000 en adelante cuando su relación con la región comenzó a intensificarse especialmente en el ámbito económico, pero con un avance paulatino hacia las dimensiones política y cultural. La comprensión de China del soft power como proceso paulatino en la primera mitad de la década de 2000 tendrá efectos medibles en la intensificación de relaciones con AL en la segunda mitad esa década. 
Joseph Nye Jr., quien en 1990 escribe su obra Bound to Lead. The Changing Nature of American Power, plantea por primera vez el concepto del soft power como un tipo de poder distinto del tradicional hard power, escribiendo "desde” y "para” los Estados Unidos (EE.UU.), como una forma de aportar teóricamente al diseño y puesta en práctica de una política exterior que le permita a dicho país perpetuar su liderazgo a escala global luego de la aparición de nuevos actores internacionales distintos al Estado nación (ONGs, Organizaciones Internacionales y actores transnacionales, entre otros), y de nuevos temas como la religión, relevante internacionalmente a partir de la revolución islámica iraní de 1979, todo lo cual, confluye con la revolución de las comunicaciones.

Para Nye Jr. el soft power es la capacidad de lograr los resultados que se desean a través de la atracción de los otros, en vez de la manipulación o coacción de ellos (2008a: 29). Se basa en la habilidad para determinar las preferencias de estos últimos -las prioridades en la agenda política internacional de un Estado, por ejemplo- para "lograr que ambicionen lo que uno ambiciona” (Nye Jr. 2003: 30). El autor aclara que el soft power no es sinónimo de influencia -aunque es una fuente de ella-, puesto que ésta puede ser parte del poder duro si se utilizan amenazas y/o pagos. Tampoco es persuasión exclusivamente, o la habilidad de mover a la gente a partir de argumentos, aunque es parte importante de esta última (Nye Jr. 2008a: 31). Las fuentes de este "poder de atracción" pueden ser: 1) su cultura (las partes de ésta que resultan atractivas para otros); 2) su política exterior (cuando ésta es vista como legítima); y 3) sus valores políticos (cuando los respeta tanto interna como externamente). Vinculado directamente con el segundo de estos recursos se encuentra un concepto clave: la diplomacia pública. Ésta puede entenderse como las políticas de comunicación de los estados que tienen por objetivo lograr el apoyo de la población de otros países a su política exterior (Noya 2005: 1). En otras palabras, es una forma de gestión del soft power por parte del Estado, a través de la elaboración de una estrategia para el uso eficiente y eficaz de los recursos de soft power que se poseen.

Sin embargo, también hay críticas al planteamiento de Nye Jr., parte de ellas sintetizadas por Javier Noya en su artículo El poder simbólico de las naciones (2005), considerando que el soft power es demasiado blando para ser un poder, o que éste último no difiere del poder duro, sino que es hard power interiorizado y convertido en ideología. El mismo Noya critica la teoría dualista de Nye Jr. al afirmar que "el soft power no es un tipo de poder, sino que cualquier recurso, incluso las capacidades militares, puede ser blando en la medida que esté socialmente legitimado para un fin” (2005: 14). En la misma línea afirma que los recursos de soft power mencionados por Nye Jr. son una serie de intangibles que el Estado no es capaz de controlar -la mayoría de ellos-, principalmente por ser éstos producidos en la economía de mercado y la sociedad civil por medios de comunicación, ONGs, empresas, etc., a diferencia de los recursos del poder duro como la fuerza militar, la que sí es controlada por el Estado. Finalmente, propone utilizar el concepto de "poder simbólico" antes que el de 
soft power, tomando en cuenta la crítica antes descrita y considerando las ideas desarrolladas por Paul Kennedy en su artículo en el Diario El País: ¿Poder duro contra soft power? de 2005, donde este último da como ejemplo la ayuda militar de EE.UU. para la reconstrucción de los países asiáticos afectados por el tsunami del 2004 como una forma de usar el poder duro con fines humanitarios, haciendo que la distinción entre hard y soft power se desvanezca.

Como uno de los aportes más recientes a su definición y desarrollo teórico, Nye Jr. (2008b) propone el concepto de smart power (o poder inteligente), que define como "la habilidad de combinar el poder duro y blando en una estrategia efectiva” (Nye Jr. 2008a: 43), dando cuenta de la complementariedad y no contraposición entre ambos tipos de poder. Esta complementariedad debería explotarse (sobre todo en la política exterior de EE.UU.) para generar soft power desde acciones que tradicionalmente se asocian al poder duro, considerando, por ejemplo, las intervenciones militares por ayuda humanitaria y las relaciones económicas con países en desarrollo.

Para desarrollar el análisis del soft power de China y su proyección hacia AL, lo haremos a través de las fuentes de este “poder de atracción” en sus dimensiones: cultural, económica, política y militar, buscando evaluar o medir la influencia de este despliegue en la intensificación de las relaciones sino-latinoamericanas en la década de 2000. Es importante mencionar que para analizar las potencias emergentes del "sur", muchas de ellas no occidentales, sin tradición democrática ni de apego a los DD.HH., es necesario tener una visión más flexible que se adapte a la realidad de dichos estados. Es el caso de China, donde los valores políticos se pueden transformar en un obstáculo o recurso contraproducente. Sin embargo, tiene cierto atractivo principalmente a través de su modelo económico y el lugar en el sistema internacional que este le ha brindado.

El análisis del artículo se estructura en dos partes: la primera, analiza cual es la visión de China sobre el soft power y la diplomacia pública en las Relaciones Internacionales; y la segunda, analiza el soft power chino en AL con especial énfasis en la década de 2000.

\section{Cómo China entiende el soft power en las Relaciones Internacionales}

La primera traducción al chino en 1992 del libro de Nye Jr. generó gran interés en el ámbito académico chino, al introducir el concepto del soft power como parte importante del poder internacional de los estados. En 1993 Wang Huning, asesor del presidente Jiang Zemin y profesor en la Universidad de Fudan, publicó un artículo en el que llama a fortalecer el soft power del país (Cho y Jeong 2008: 456) agregando además que la cultura china debería ser su principal recurso (Glaser y Murphy 2009: 11). 
A partir de este trabajo se desarrollarán otros como el de Pang Zhongying en 1997 en el que introduce la teoría de Nye Jr. con gran detalle, o el de Shen Jiru en 1999 en el que al igual que Huning aboga por una estrategia que tenga por objeto aumentar el soft power de China (Cho y Jeong 2008: 456).

Sin embargo, la discusión tomará mayor intensidad desde el inicio de la "era Hu Jintao" en 2004, momento en que el concepto comienza a ser incorporado por las autoridades del gigante asiático, luego de años de concentración en el poder -duro- económico y militar tras las reformas económicas de 1978. Con la llegada de $\mathrm{Hu}$ al poder el soft power se introducirá de forma explícita en el discurso del gobierno chino (Glaser y Murphy 2009: 15-16; Cho y Jeong 2008: 459) y en su política exterior, a través de la diplomacia pública -otro concepto nuevo en China, y que se irá desarrollando paralelamente al de soft power- como una forma de lograr un poder comprehensivo que combine hard y soft power, además de servir como instrumento para mejorar la imagen del país en el exterior. Esto último adquiere sentido considerando el contexto posguerra fría en el que su emergencia como potencia regional y global comienza a consolidarse en los ámbitos económico (con tasas de crecimiento económico por sobre el 8\% sostenidas por más de 20 años) y político (con una participación activa en el Consejo de Seguridad de la ONU), incorporándose además de forma definitiva a la comunidad internacional y a diversos espacios multilaterales como la ONU y la OMC (esta última en 2001), quedando China expuesta a críticas por su sistema político y económico.

Frente al auge del gigante asiático desde sus reformas de apertura económica a fines de los 70' se evidenció un aumento del temor en su región, en la Unión Europea (UE) y en EE.UU. sobre un posible ascenso similar al de la Alemania nazi, el Japón imperial y la URSS de Breznev del siglo XX, desarrollándose diversas teorías como la de "la amenaza china", según la cual el despertar de China podría generar conflictos mundiales o regionales, bloques militares o carreras armamentistas (Bustelo 2005: 2). Parte importante de estas teorías se enmarcan dentro de la corriente realista de la disciplina de las Relaciones Internacionales, incorporando su lógica de juego de suma cero en la que China buscaría establecer una hegemonía o control exclusivo de Asia (Niu 2011: 2). Como respuesta a estas percepciones y teorías, el gobierno chino comenzará a fortalecer su soft power a partir de una política exterior multidimensional con un uso efectivo de su diplomacia pública, presentando a China como una potencia responsable y sin pretensiones hegemónicas, cooperativa y promotora de la paz internacional (Rocha 2006: 706).

Dentro de esta nueva perspectiva, dos conceptos serán centrales: el "ascenso pacífico" y el "mundo armonioso". La teoría del ascenso pacífico de China fue desarrollada por académicos y especialistas chinos en relaciones internacionales, apoyados por el gobierno de Hu Jintao y por la dirección del Partido Comunista Chino (PCCh). Su principal objetivo consistió en dar respuesta a teorías como la antes mencionada sobre la "amenaza china”, difundidas principalmente en ciertos círculos académicos y think 
tank del mundo occidental y Japón en Asia oriental (Rocha 2006: 706). Este enfoque se asocia principalmente a Zheng Bijan, vicepresidente ejecutivo de la Escuela Central del PCCh entre 1993 y 2002 -presidiendo luego un think tank cercano a Hu Jintao- e introductor de esta noción según la cual China se presenta como una potencia emergente pero responsable, un "poder pacífico, no amenazante, pero sin renunciar a los derechos y responsabilidades que le aguardan por su nueva posición en el mundo" (Ibid: 706).

En el año 2004 Hu Jintao decide reemplazar la palabra "ascenso" por "desarrollo", en la medida que el primer concepto podría sugerir una intensión china de romper el statu quo internacional (Cho y Jeong 2008: 467). De este modo comienza a hablarse del "desarrollo pacífico de China”, doctrina explicada de la siguiente forma en el Libro Blanco China's Path to Peaceful Development publicado por el gobierno chino en 2005 (Ibid: 468): primero, el desarrollo pacífico es un camino que China tomará inevitablemente en su proceso de modernización; segundo, China logra este desarrollo creando un ambiente global pacífico y facilitando la paz mundial a través del mismo; tercero, China logra su desarrollo confiando en sus propias capacidades, como en la reforma e innovación; cuarto, China se acomoda a las tendencias de la globalización y se esfuerza por lograr beneficios mutuos y desarrollo común con otros países; quinto, China adhiere a los principios de paz, desarrollo y cooperación, y lucha por la construcción de un mundo armonioso sustentado en la paz y prosperidad común. Estos principios dan cuenta del rol que China pretende jugar en el sistema internacional, dejando en claro que el país "puede y quiere ascender sin poner en cuestión, desafiar o incluso perturbar el orden internacional existente” (Bustelo 2005: 4).

Además del “desarrollo pacífico”, el segundo concepto clave que se ha utilizado desde el gobierno chino para eliminar la suspicacia y temor del auge de China es el del "mundo armonioso". Esta idea tiene su origen en la filosofía de Confucio, quien expuso que a pesar de que el mundo está lleno de diferencias y contradicciones, el hombre honrado debe equilibrarlas y conseguir la armonía (Spanish.china.org.cn 2007). La política del "mundo armonioso" se basa justamente en este principio, y se sustenta en cinco elementos (Youming 2010: 1) una estrategia ganar-ganar (win-win); 2) el desarrollo pacífico; 3) el respeto a la diversidad; 4) la cooperación y coordinación; y 5) la coexistencia pacífica. Ambos conceptos -el desarrollo pacífico y el mundo armonioso- han articulado el discurso del gobierno chino en lo que algunos autores han denominado como la "ofensiva de encantamiento" o "charm offensive" (Kalathil 2011: 1; Glaser y Murphy 2009: 11), cuya finalidad es mejorar la imagen de China en el exterior, eliminando prejuicios y temores que le impidan al gigante asiático consolidarse como una potencia regional y global responsable y confiable.

En suma, será a partir de la década del 2000 en que se incorporará el soft power de forma explícita en el debate académico y en la política exterior china como uno de sus pilares fundamentales. Esto da cuenta de un 
cambio en el actuar del gobierno chino internacionalmente, comprendiendo que para lograr un desarrollo económico sostenido necesita de un ambiente externo pacífico que vea en China no una amenaza, sino una fuerza benigna, cooperativa y dispuesta a participar responsablemente dentro de la comunidad internacional. Un contexto de estas características le facilitará a China el mantenimiento de sus relaciones económicas, políticas y culturales a lo largo y ancho del orbe, vínculos que le han permitido responder a las demandas internas relativas a su crecimiento demográfico y la modernización del país (le han posibilitado importar gran cantidad de materias primas y exportar bienes manufacturados, algo que seguirá necesitando en los próximos años), además de lograr un apoyo significativo de otros estados de la comunidad internacional en temas tan delicados como el de Taiwán y la postura de Beijing de "Una sola China” (Kalathil 2011: 4).

\section{Dimensiones del soft power chino: proyecciones y contradicciones para América Latina}

Desde el 2000 en adelante, identificamos una nueva etapa de las relaciones sino-latinoamericanas ya caracterizada por una intensificación de la relación ya no sólo en términos económicos, si no que también en su dimensión política con avances en la formalización de relaciones diplomáticas con nuevos estados de AL que reconocen a China, siendo el más relevante Costa Rica en 2007 con quien firma un tratado de libre comercio el 2010, y también con el aumento de los contactos político/diplomáticos, incluyendo las visitas del entonces presidente chino Jiang Zemin en 2001, el primer ministro Wen Jiabao en 2003 y 2012, y el actual presidente Hu Jintao en 2004 y 2005, lo anterior reforzado por el vínculo cultural, con la firma de diversos acuerdos en la materia, y el aumento de intercambios culturales y eventos artísticos. En el año 2008, China hizo un intento por transparentar su creciente interés en la región lanzando un "Documento Oficial sobre la Política de China hacia América Latina y el Caribe" (Shixue 2011: 59), dando cuenta de la importancia que ha adquirido AL en la política exterior de Beijing. Finalmente, se debe mencionar el vínculo político establecido con organizaciones regionales tales como el Grupo de Río, Mercosur, la OEA, la CEPAL, ALADI y el BID, además del antiguo existente entre el PCCh y algunos partidos políticos latinoamericanos (Lee 2011: 47-48).

En esta nueva etapa de las relaciones sino-latinoamericanas desde el 2000 es posible analizar el uso del soft power por parte de China hacia AL utilizando principalmente cuatro dimensiones de su política exterior: en primer lugar, la dimensión cultural donde el aspecto más estratégico es la instauración de numerosos Institutos Confucio en varios países de la región; en segundo lugar, la dimensión política que ha potenciado la buena evaluación del modelo de desarrollo chino; en tercer lugar, la dimensión económica que refuerza los lazos de la cooperación sur-sur, y por último, la dimensión militar con énfasis en la cooperación tecnológica y las operaciones de paz. 
4.1 Dimensión Cultural. La cultura china es considerada por diversos autores como el principal recurso de soft power del gigante asiático (Lee 2011: 31; Cho y Jeong 2008: 470; Glaser y Murphy 2009: 13), debido a la atracción que por milenios ha generado en el mundo su complejidad y riqueza filosófica. En el centro de ésta se encuentra el Confucianismo, antigua filosofía centrada en Confucio (551-479 a.C.) fundadora de las bases sociopolíticas de China, a partir de principios como el respeto de la familia en tanto núcleo de la sociedad, la preocupación por las virtudes y la ética, la primacía del grupo sobre el individuo, el énfasis en la unidad, la armonía y el orden, la importancia del trabajo y la educación, entre otros (Cho y Jeong 2008: 470). Estas ideas se han incorporado también en las orientaciones de la política exterior actual china, en la medida que se buscaría la armonía en el mundo, el orden y la paz internacional, a través de un soft power "basado en los valores confucianos tales como la modestia, la tolerancia y la disposición a aprender” (Lee 2011: 33).

Desde el gobierno de Hu Jintao -y también antes, pero en menor medida- se ha tomado conciencia de la importancia de proyectar la cultura china en el mundo como el principal recurso de soft power del país, considerando la atracción que ésta genera. Para lograr dicho objetivo China ha desarrollado su diplomacia pública que consiste en programas del gobierno cuyo objetivo es informar o influir en la opinión pública de otros países, teniendo como principales instrumentos las publicaciones, películas, intercambios culturales, radio, televisión, turismo y deporte, además de la promoción del idioma y la celebración de conferencias, simposios y talleres en el extranjero (Saddiki 2009: 109).

Una de sus principales iniciativas ha sido el establecimiento de Institutos Confucio a lo largo y ancho del orbe, instalándose el primero en Seúl el año 2004 y aumentando vertiginosamente en número hasta llegar a ser 353 Institutos y 473 Aulas Confucio en 2012 (Ren 2012: 2). Son escuelas establecidas por el gobierno (en específico la Office of Chinese Language Council International, o Hanban por su abreviación en chino) en cooperación con universidades extranjeras e instituciones educacionales, cuyo objetivo es promover la comprensión de la lengua y cultura china (Ibid: 1). Esta política ha tenido un gran éxito en un corto periodo de tiempo, inscribiéndose miles de alumnos en sus primeros años de funcionamiento. Además, el estudio del idioma chino se ha incorporado en más de 2.500 universidades del mundo, con más de 40 millones de estudiantes (Ibid: 3). También la realización de los Juegos Olímpicos en Beijing el año 2008 y la Expo Shanghai en 2010, que le dieron a China una oportunidad única para presentar la grandeza de su país, promoviendo la "Marca China” (Wang 2008: 264). El cine chino también gana en volumen (330 películas producidas en China en 2006) y premios, como el León de Oro de Venecia otorgado a Jia Zhangke por su filme Still Life en 2006, un ejemplo del lento pero creciente progreso de éste en las taquillas occidentales (Roth 2007: 5), además del Premio Nobel de la Paz en 2011 y el Premio Nobel de Literatura en 2012. 
En AL también es posible constatar este despliegue de soft power a través de la variable cultural en la segunda mitad de la década de 2000, considerando que el año 2006 se establece el primer Instituto Confucio en México y hoy tenemos 25 Institutos Confucio y 10 Aulas Confucio en 12 países de América Latina (Spanish.hanban.org 2012), destacando: México (4), Perú (5), Colombia (2), Cuba (1), Chile (2), Brasil (3), Argentina (2), Costa Rica (1).

Otro de los aspectos que se consideran dentro de la diplomacia cultural son los medios de comunicación utilizados por el gobierno para promover su soft power. En el caso de China existen diversos ejemplos, entre los que destacan el canal de televisión a 24hrs CCTV 9, que comenzó a emitir en 2007 y que para 2009 tenía servicios en inglés, español, francés, ruso y árabe, además de la agencia Xinhua, la People’s Daily y el proyecto reciente Global Times que pretende competir internacionalmente con CNN, BBC o Al-Jazeera (Zai China 2010).

El gobierno chino se ha preocupado además de ampliar su influencia mediática en AL, aumentando el apoyo a empresas editoriales y medios de comunicación de dicha región (Observatorio de la política china 2012). Según el informe "Cómo China persigue la influencia mediática en África, América Latina y el Sudeste Asiático”, publicado en 2010 por el CIMA (Centro para la Asistencia Internacional de los Medios), los intereses de los medios de comunicación chinos en AL se han centrado en Venezuela, Nicaragua, Bolivia y Ecuador (Radio Nederland Wereldomroep Latinoamerica 2012), lo que no significa que no tenga presencia en el resto de la región, sobre todo considerando la gran cantidad de sitios webs, revistas, prensa (escrita y online) y canales de tv que pueden ser visitados desde AL.

Por último, un factor que influye en la proyección de la cultura china en el mundo pero que no es controlado por el gobierno chino directamente es la diáspora china, calculada en 50 millones (Guerra 2010: 2) de “embajadores” culturales dispersos en todas las regiones del mundo, estando mayormente concentrados en el Sudeste Asiático y en Norteamérica y Australia. En AL la población proveniente de China comenzó a incrementarse desde los 90' en adelante, en parte debido a una relativa apertura en las políticas migratorias chinas y de una concomitante diversificación de los destinos de dichas migraciones, sin representar de todas formas un gran número respecto de la población total de los países destino -salvo en el caso de Panamá, en donde representan un 12\% de los extranjeros- (Courtis 2012: 2). Siguiendo los datos de Ellis (2009), quien da cuenta de la dificultad que existe para medir con certeza la cantidad de migrantes chinos en AL, y la precaución que se debe tener para hacer generalizaciones al respecto, podemos constatar que las comunidades chinas se han concentrado mayormente en Brasil (300 mil habitantes en 2005), Panamá (150 mil) y Venezuela (130 mil), seguidos por Argentina (65 a $70 \mathrm{mil}$ ), Costa Rica (60 mil), Ecuador (50 mil), México (31 mil), Bolivia (12 mil) y Chile (7 mil). Respecto de Perú, se señala que no existe una cifra oficial -hasta 2009 al menos- pero existen estudios que señalan que de 4,2 millones de 
peruanos -un 15\% de la población- tendrían alguna descendencia china.

4.2 Dimensión Política. Además de un proceso de acercamiento político de China a AL desde la década del setenta en adelante, que incluye el reconocimiento diplomático de los países de la región, hay también un acercamiento más reciente que se ha desplegado durante la década de 2000 y que enfatiza la segunda mitad de esta década coincidiendo con la incorporación del soft power en la política exterior hacia AL. Esta asertiva estrategia para desarrollar el vínculo político con la región se puede constatar en dos aspectos: en primer lugar, la buena evaluación de los países latinoamericanos del modelo de desarrollo chino que se complementa con el principio de no intervención en los asuntos internos, y en segundo lugar, la sintonía con los cambios que mueve China en el sistema internacional buscando una multipolaridad en beneficio de los países en desarrollo.

Con respecto al modelo de desarrollo, visto desde Occidente -entendido como Europa, EE.UU., y en algunos casos AL-, la ausencia de un sistema democrático, las violaciones a los DD.HH., la censura en los medios, la persecución política a líderes opositores del régimen como fue el caso del premio Nobel de la Paz 2010 Liu Xiaobo, además del apoyo del gigante asiático a regímenes autoritarios como el de Myanmar o Corea del Norte, son aspectos que afectan de forma negativa a la imagen de China en el mundo, y por lo mismo, a su soft power. Esto último principalmente por la contradicción que existiría entre la imagen que busca proyectar al exterior, y lo que realmente realiza dentro y fuera de sus fronteras, algo que podría terminar alejando a China de su objetivo de ser vista como un actor internacional responsable (Kalathil 2011: 8).

Sin embargo, lo anterior es contrarrestado en los países en desarrollo por la percepción positiva que tienen del modelo económico chino el cual integra al autoritarismo político como parte de su funcionamiento. Este "estilo chino de socialismo" (Cho y Jeong 2008: 465) emerge entonces como una alternativa al modelo occidental para alcanzar el desarrollo, sobre todo en el contexto internacional de crisis financiera desde el año 2008 en adelante. Este modelo también denominado "Consenso de Beijing”, combina los siguientes elementos según el análisis de Fanjul (2009: 2-6): un capitalismo de Estado, en el que este último ejerce un rol central en el sistema económico, ya sea por medio de empresas públicas como por intervenciones directas en él; un gradualismo en la política de reformas, estrategia distinta a la ocupada por Europa del Este tras la caída del muro de Berlín, caracterizada esta última por aperturas liberalizadoras bruscas y privatizaciones masivas. El gradualismo da cuenta de un valor político y filosófico propio de China, la importancia de la prudencia, los cambios paulatinos; un modelo abierto al exterior, proceso que se ha desarrollado de forma gradual, acorde con lo antes mencionado; el autoritarismo político, con un PCCh a la cabeza que cuenta con un importante grado de legitimidad (tanto por su rol en la historia de China, como por los logros económicos del país), y un sistema caracterizado por los aspectos mencionados en el párrafo anterior, autoritarismo que convive con la revolución eco- 
nómica; y una gran capacidad de flexibilidad y adaptación ante nuevas circunstancias, aspecto clave en el éxito económico del gigante asiático.

La atracción de este modelo se relaciona directamente con la forma exitosa en que China ha enfrentado la crisis financiera mundial, entrando tarde a ella y siendo posiblemente uno de los primeros países del mundo en lograr salir de la misma (Fanjul 2009: 1-2), en contraste con la ineficacia que han demostrado las democracias occidentales en dicha empresa. En cuanto a la posibilidad de “exportación” de este modelo, el gobierno chino ha sido muy cauto en señalar que representan una alternativa a Occidente pero que no pretenden competir, mucho menos con EE.UU. (Glaser y Murphy 2009: 23), especificando que otros países pueden tomar algunas partes de su modelo para crear sus propios modelos de desarrollo de acuerdo a su particularidades propias y no tomar el modelo de forma íntegra.

De este modo podemos identificar valores y principios políticos propios de China que pueden ser considerados atractivos en otras partes del mundo -sobre todo para los países en desarrollo-, tanto en su modelo de desarrollo como en su actuar internacional, a través de la defensa del multilateralismo, la justicia, la paz, la multipolaridad y la búsqueda de armonía en las relaciones internacionales (Lee 2011: 41-43), aspecto que se ha reforzado a favor de China por el contexto de crisis económica en EE.UU. y Europa desde el 2008.

4.3 Dimensión Económica. Dentro del análisis original de Nyr Jr. respecto de las fuentes del soft power, la dimensión económica quedaba fuera al relacionarse directamente con el poder duro clásico. Sin embargo, para analizar el soft power chino utilizaremos una conceptualización más flexible tomando algunos aspectos económicos como la cooperación internacional, el comercio y la inversión en diversos países como un recurso válido de soft power en la medida que mejora la imagen de China en los lugares donde realiza estas acciones. Como menciona Wuthnow (2008: 16), este tipo de cooperación o ayuda puede considerarse como una especie de “diplomacia económica”, que tendría como objetivo promover relaciones positivas con los países en desarrollo, además de reducir la animosidad o suspicacia respecto del auge de China.

China en particular posee su enclave en términos de Ayuda al Desarrollo a través de la Cooperación Sur-Sur y su finalidad es la obtención de materia prima a cambio de inversión en infraestructura, estadios, aeropuertos, entre otros, lo que a su vez se propone asegurar o ganar el reconocimiento diplomático de Una China frente a Taiwán. Esta diplomacia económica se ha focalizado en el Sudeste Asiático (SEA), África y AL principalmente, y se caracteriza por no exigir -en la mayoría de los casos- condiciones que otros donantes sí -como la UE o EE.UU.-, como reformas democráticas, apertura de mercado y protecciones medio ambientales (Lum et al. 2008: 4), además de entregar préstamos con bajas tasas de interés y perdonar deudas. 
En este contexto de diplomacia económica, China desde 1993 ha establecido “Asociaciones Estratégicas” con diversos países a nivel mundial, dicho estatus en la política exterior de China implica además de reconocimiento el establecimiento de mecanismo de coordinación y resolución de conflictos ya sea en el ámbito económico o político. Sin duda, el requisito principal para obtener este estatus es ser una potencia regional influyente en la toma de decisiones en instituciones regionales o en sobre el resto de los países de la región.

Este soft power a través de alianzas económicas también se expresa en AL con mayor intensidad durante la década de 2000. Como plantea Eduardo Oviedo (2006: 385), desde 1993 la RPCH plantea en muchos tratados y comunicados con algunos miembros de la comunidad internacional, la idea de relaciones estratégicas y destacamos aquí que Brasil es el único país de la región latinoamericana de tener este estatus el mismo año 1993, los otros países de la región que lo tienen en la década de 2000, Venezuela (2001), Argentina (2004), Perú (2008), y Chile (2012). Según plantea el académico, la estrategia tiene un sentido o significancia muy particular en el contexto chino actual, ya que no se traza desde una alianza militar sino de una alianza asociativa o de "socios", tomando a la economía como eje central de las relaciones. Como explica Fernando Delage, director del centro de Casa Asia en Madrid:

“se debe tener en cuenta que China está desarrollando intereses es-
tratégicos globales y, por tanto, depende del sistema internacional.
Pekín está concentrándose principalmente en su modernización eco-
nómica y militar, en evitar conflictos innecesarios y en ganar presti-
gio y poder internacional. Si bien China puede sentirse incómoda
con la idea de un orden mundial dominado por Estados Unidos; no
intentará transformarlo mediante el uso de la fuerza: un sistema in-
ternacional estable es la condición indispensable para asegurar su
crecimiento así como un mayor status diplomático" (Límberg 2009: 8).

4.4 Dimensión Militar. Siguiendo con la conceptualización flexible respecto de los recursos de soft power, consideraremos un aspecto específico de la fuerza militar que puede contribuir a mejorar la imagen de China en el mundo: la cooperación tecnológica y sus Operaciones de Paz. En este sentido, la participación del gigante asiático en Operaciones de Paz de la ONU en Sudán, el Sahara occidental, Costa de Marfil, Etiopía, Liberia y en la República Democrática del Congo, también contribuirían a fortalecer el soft power chino, puesto que le otorgaría buena reputación a nivel internacional (Struye de Swielande 2009: 11). Además, el aumento sostenido en la contribución de tropas para misiones de paz le permitiría responder a las inquietudes -de EE.UU. por ejemplo- respecto del rol que jugará en la comunidad internacional -compromiso y responsabilidad- (Wuthnow 2008: 20).

En AL la cooperación militar con China se ha desarrollado de forma paralela a las otras dimensiones de la relación sino-latinoamericana, inten- 
sificándose principalmente desde la era Hu Jintao y su visita a AL en 2004. En dicho año se realizaron 20 visitas de oficiales chinos de defensa a AL y el Caribe, y viajaron ministros y Jefes de Defensa de nueve países de la región a China (Domínguez 2006: 6), tendencia que se mantiene constante hasta hoy (Ellis 2011: 16).

Asimismo, la cooperación se ha desarrollado en gran medida en áreas estratégicas, principalmente a partir de la transferencia de tecnología aeronáutica, satelital y de telecomunicaciones, destacando el caso de Argentina y su acuerdo para la venta de reactores nucleares de 2004, y el de Brasil y su cooperación en materia satelital (desde los 90' con cinco acuerdos entre 1998 y 2004), la que tuvo como resultado el lanzamiento en 1999 del Satélite para Exploración de los Recursos terrestres Sino-Brasilero y en 2003 el cohete Larga Marcha 4B (Malena 2007: 1-3). También se puede destacar a Venezuela y la fabricación y lanzamiento en conjunto con China del primer satélite artificial venezolano en 2008 -Venesat I o Simón Bolívar- a partir de un acuerdo firmado en 2005 con Beijing (Ríos, 2009: 3). Otro ejemplo son los casos de EMBRAER de Brasil y la China Aviation Industrial Corporation (CAIC), que colaboran para producir los ERJ-145 en Harbin, China, como también colaboran para vender aviones turbofan Y12 a Venezuela en el marco de la creación de una aerolínea regional (Ellis 2011: 33).

Por último, respecto de las Operaciones de Paz es relevante mencionar que China participa en forma conjunta con países de AL y el Caribe en la Misión de Estabilización de las Naciones Unidas en Haití (MINUSTAH), establecida en 2004 y que en la actualidad incluye las contribuciones latinoamericanas militares y/o de agentes policiales de Argentina, Bolivia, Brasil, Chile, Colombia, Ecuador, El Salvador, Paraguay, Perú y Uruguay, entre otros (Rodríguez 2012: 47). Esta presencia física de China en AL también se constata en los ejercicios militares conjuntos que se realizaron en Perú en noviembre de 2010, en el marco de asistencia humanitaria, operación que se denominó Angel de la Paz. También en Cuba, China tiene presencia física en tres bases de la era soviética, Lourdes, Bejucal y Santiago de Cuba, con énfasis en espionaje electrónico y computacional así como actividades de intercepción de radios y comunicación. Por otra parte, destaca la presencia de China en la Antártica a través de bases de investigación científicas desde 1985 en las cuales también hay colaboración del ELP desde el año 2004, lo que de paso, se transforma también en una instancia de colaboración con otras bases, por ejemplo, la base chilena que por su proximidad permite interacciones con militares chilenos (Ellis 2011: 32-36).

\section{Conclusiones}

El artículo analiza el soft power de China en América Latina constando dos ideas: por un lado, el concepto de soft power se incorpora al discurso internacional de China desde el año 2004 durante el gobierno de Hu Jintao, y segundo, dicha incorporación tiene consecuencias para América Latina en cuanto es posible medir sus efectos en la segunda mitad de la 
década de 2000 en lo cultural, político, económico y militar.

En Relaciones Internacionales el concepto de soft power surge en 1990 acuñado por Nye Jr., entendido como la capacidad de lograr los resultados que se desean a través de la atracción de los otros, en vez de la manipulación o coacción de ellos. Los principales recursos de soft power que señala Nye Jr. son la cultura, los valores políticos y la política exterior de un Estado, en la medida que éstos sean "atractivos” para otros. De esta forma, se estableció como un tipo de poder distinto del poder duro clásico, militar y económico, diferenciación que, sin embargo, ha sido criticada desde su origen.

Para analizar a China utilizamos esta conceptualización de Nye Jr. pero de manera flexible, tomando a la política exterior como un marco amplio que incluye una dimensión cultural, una económica, una política y una militar, y considerando que, como menciona Nye Jr., en la práctica cualquier recurso que sea atractivo para otros estados puede beneficiar al soft power del país que lo utiliza. Sostenemos en el análisis, que el soft power no se define simplemente por el uso de recursos intangibles, la forma en que se utilizan los recursos y el resultado que estos generen son aspectos necesarios de considerar como marco analítico para identificar la presencia del soft power de China en América Latina en las esferas económica y la militar, las cuales poseen aristas que demuestran que no solo deben ser encasilladas en la categoría hard power. En este sentido, constatamos que China ha dedicado más su energía al crecimiento económico que al fortalecimiento del soft power en materia de atracción cultural y valórica, siendo la atracción de su éxito económico la fortaleza esencial en el mundo y en América Latina en particular según nuestro análisis.

Además de este ajuste analítico del concepto, es necesario considerar que el concepto de soft power surge "desde" y "para” EE.UU. y, por lo mismo, no puede aplicarse de igual forma en el gigante asiático, en cuanto este último carece de una característica fundamental en el soft power norteamericano: la tradición democrática. No obstante, China ha desplegado su influencia a través de su modelo de desarrollo el que integra naturalmente la variable del autoritarismo político. Así, la configuración de una imagen ambivalente para los países en desarrollo es una estrategia que hasta el momento ha tenido buenos resultados por parte de China al dirigir una política de cooperación para el desarrollo del sur, transmitiendo un perfil fraternal y de líder simultáneamente. El resultado, los países de América Latina valoran el modelo de desarrollo chino independiente de la variable política, más aún en un contexto internacional marcado por los desequilibrios económicos y financieros de las tradicionales potencias económicas del sistema internacional desde el 2008 hasta hoy.

En consecuencia, China cuenta con soft power y lo ha incorporado en su discurso y política exterior, sobre todo desde la "Era Hu Jintao" 2004- 2012-, proyectándolo al mundo a partir de su política de "ascenso pacífico" -luego redefinido como "desarrollo pacífico"-, que tiene por ob- 
jetivo disipar los temores respecto del auge de China, y fortalecer su soft power a través de una política exterior basada en sus dimensiones cultural (estableciendo Institutos Confucio en el mundo, y realizando intercambios culturales y exposiciones, entre otras acciones), económica (a partir de cooperación e inversión en infraestructura), política (presentando al "Consenso de Beijing” como modelo de desarrollo alternativo al de Occidente, por ejemplo) y militar (participando en Operaciones de Paz de la ONU, dando cuenta de su compromiso con la comunidad internacional).

Asimismo, de seguir aplicando esta política exterior de "encantamiento" y búsqueda de un "mundo armonioso" a partir de su "desarrollo pacífico”, lo más probable es que su estrategia de soft power se haga cada vez más efectiva en las regiones del mundo que considera estratégicas. Para lograr esto último aún le quedan desafíos importantes, sobre todo en lo relativo a su sistema político autoritario y las violaciones a los DD.HH., además de sus vínculos con gobiernos dictatoriales como Myanmar o Corea del norte, todos estos factores que afectan de forma negativa a la imagen de China en Occidente. No obstante, hasta ahora su discurso e ideas por cambiar el sistema internacional a favor de los países en desarrollo en una estructura multipolar han sido inclusivos de la diversidad de países en sus sistemas económicos y políticos. La no intervención en los asuntos internos es para China pieza fundamental de su política exterior y es un aspecto que flexibiliza su relación con el sistema internacional.

Las consecuencias de lo anterior para América Latina son constatables en el marco de la década de 2000, en la cual se inicia una nueva etapa en las relaciones sino-latinoamericanas ya que desde entonces se registran relaciones permanentes y de mayor frecuencia en los variados aspectos. En el contexto de esta nueva etapa es posible argumentar que el interés de China por desarrollar más estrechas relaciones con América Latina se enmarca en un proceso de transformación de la política exterior de la potencia asiática en cuanto incorpora la variable del soft power en su comprensión de las Relaciones Internacionales, a la vez que despliega su diplomacia pública a regiones de países en desarrollo que necesitan cooperar para insertarse y transformar el sistema internacional a su favor.

$\mathrm{Al}$ respecto, identificamos cuatro dimensiones de la política exterior china que nos permitieron identificar los aspectos claves del despliegue de su soft power: en primer lugar, la dimensión cultural que se centra en instalar numerosos Institutos Confucio en varios países de la región después del año 2006 siendo actualmente 12 países favorecidos sumando un total de 25 institutos en la región; en segundo lugar, la dimensión política que ha potenciado la buena evaluación del modelo de desarrollo chino que China complementa con un discurso internacional de no intervención en los asuntos internos de los países, y esto nos lleva a la tercera dimensión, la económica, donde China despliega una política de hermandad y solidaridad que a través de una cooperación sur-sur promociona su crecimiento como un modelo chino de exportación a través de los postulados del Consenso de Pekín en cuanto a una apertura económica gradual y controlada 
permitiéndole consolidar vínculos para el acceso a materias primas en pos de su progreso industrial y tecnológico; y por último, la dimensión militar con énfasis en la cooperación tecnológica aeronáutica, satelital y de telecomunicaciones y en las operaciones de paz que le permite a China levantar otras aristas de soft power dentro de una capacidad clásica del hard power, el resultado es mayor presencia física de China en América Latina. Estas es una clara demostración de cómo el soft power necesita ser analizado en función de la complementariedad que existe con el hard power, emergiendo la configuración de un poder inteligente, capaz de utilizar estratégicamente ambas capacidades según el contexto, que en la política exterior de China se entiende como pragmatismo. 


\section{Nota}

${ }^{1}$ Este artículo es parte del Proyecto de Investigación $N^{\circ} 11100096$ financiado por el Fondo Nacional de Desarrollo Científico y Tecnológico (FONDECYT) de Chile. 


\section{Bibliografía}

Bustelo, Pablo (2005), "El Auge de China: ¿amenaza o "ascenso pacífico?”, en Real Instituto Elcano de Estudios Internacionales y Estratégicos, núm. 135, Madrid.

Cho, Young y Jeong, Jong (2008), “China’s Soft Power: Discussions, Resources, and Prospects”, en Asian Survey, vol. 48, núm. 3, Estados Unidos: University of California Press.

Courtis, Corina (2012), “Migración china en el sur de América Latina: una aproximación socio-demográfica a partir de datos censales de Argentina y Chile”, Argentina: Universidad de Buenos Aires.

Domínguez, Jorge (2006), "China’s Relations With Latin America: Shared Gains, Asymetric Hopes”, en Inter-American Dialogue, Estados Unidos: Harvard University.

Ellis, Evan (2009), China in Latin America: The Whats and Wherefores, Estados Unidos: Lynne Rienner Publichers, inc.

Ídem (2011): China-Latin America military engagement; good will, good business, and strategic position. EE.UU. Estados Unidos: Strategic Studies Institute (SSI). En http://www.StrategicStudiesInstitute.army.mil/

Fanjul, Enrique (2009), "El Consenso de Pekín: ¿un nuevo modelo para los países en desarrollo?”, en Real Instituto Elcano de Estudios Internacionales y Estratégicos, núm. 122, Madrid.

Glaser, Bonnie y Murphy, Melissa (2009), "Soft Power with Chinese Characteristics: The ongoing debate", en McGiffert, Carola et al. [comps.], Chinese Soft Power and Its Implications for the United States, USA: Center for Strategic \& International Studies.

Guerra, Carolina (2010), “La Diáspora China”, en Consejo Argentino para las Relaciones Internacionales, núm. 6, Buenos Aires.

Jiru, Shen (1999), “Can’t neglect strengthening our country's soft power”, en Outlook Weekly, núm. 41.

Kalathil, Shanthi (2011), “China's Soft Power in the Information Age: Think Again”, en Institute fot the Study of Diplomacy, Estados Unidos: Georgetown University.

Kennedy, Paul (2005), “¿Poder duro contra soft power?”, en El País, España. Disponible en: <http://elpais.com/diario/2005/02/19/opinion/ 1108767607_850215.html> [2 de septiembre de 2012].

Lee, Yun (2011), “Obstáculos y desafíos al surgimiento del soft power de 
China en América Latina”, en Lee, Yun y Wu, Hong [comps.], Chile y China, Cuarenta años de Política Exterior, Santiago: RIL Editores.

Límberg, Chero (2009), “Las relaciones políticas y económicas entre China y Perú 1998-2008”, Perú: PLADES.

Lum, T., et al. (2008). China's “Soft Power” in Southeast Asia. Congressional Research Service, USA.

Malena, Jorge (2007), “La cooperación militar entre China y América Latina: realidad o mito?”, en Observatorio de la política china. Disponible en: <http://www.politica-china.org/nova.php?id=98\&clase=6\&lg=gal $>$ [9 de agosto de 2012].

Misión de Estabilización de las Naciones Unidas en Haití (MINUSTAH): http://www.un.org/es/peacekeeping/missions/minustah/

Niu, Xinchun (2011), "Eight Myths about Sino-U.S. Relations”, en Contemporary International Relations, núm. 21, China.

Noya, Javier (2005). El Poder Simbólico de las Naciones. Real Instituto Elcano de Estudios Internacionales y Estratégicos, $\mathrm{N}^{\circ} 35$.

Nye Jr., Joseph (2008a), The powers to Lead, USA: Oxford University Press.

Ídem (2008b), “Smart Power and the 'War on Terror'”, en Asia-Pacific Review, vol. 15, núm. 1, Estados Unidos.

Ídem (2003), La paradoja del poder norteamericano, Madrid: Taurus.

Observatorio de la política china (2012), "Medios de comunicación chinos procuran mejor ubicación en el extranjero”. Disponible en: <http:// www.politica-china.org/nova.php?id=2684\&clase=8\&lg=gal $>$ [8 de agosto de 2012].

Oviedo, Eduardo (2006), “China: visión y práctica de sus llamadas ‘relaciones estratégicas””, Estudios de Asia y Africa, Vol. XLI, N 3, El Colegio de México, México.

Radio Nederland Wereldomroep Latinoamérica (2012), “América Latina y la influencia mediática china”, Holanda. Disponible en: Recuperado el 8 de agosto de 2012: <http://www.rnw.nl/espanol/article/am\%C3\%A9ricalatina-y-la-influencia-medi\%C3\%A1tica-china> [8 de agosto de 2012].

Ren, Zhe (2012), “The Confucius Institutes and China’s Soft Power”, en Institute of Developing Economies, núm. 330, Japón.

Rocha, Manuel (2006), “China en transformación: la doctrina del desarro- 
llo pacífico”, en Foro Internacional, vol. 46, núm. 4, México: El Colegio de México.

Rodríguez, Isabel (2012): Convergencia de Intereses Políticos y Estratégicos entre China y América Latina: periodo 2000-2010. Escenarios Actuales, CESIM, N² 2, Septiembre, 2012.

Roth, Jaime (2007), “La nueva diplomacia cultural china”, en Real Instituto Elcano de Estudios Internacionales y Estratégicos, núm. 103, Madrid.

Saddiki, Said (2009), "El papel de la diplomacia cultural en las relaciones internacionales”, en Revista CIDOB d'Afers internationals, núm. 88, Barcelona.

Shixue, Jiang (2011), “Ten Key Questions”, en Hearn, Adrian y LeónManríquez, José Luis [comps.], China Engages Latin America: Tracing the Trayectory, USA: Lynne Rienner Publishers, Inc.

Spanish.china.org.cn (2007). "Un mundo armonioso: Un antiguo concepto filosófico chino para el nuevo orden internacional”. Disponible en: <http:/ /spanish.china.org.cn/china/archive/shiqida/2007-10/08/ content_9012085.htm> [18 de junio de 2012].

Spanish.hanban.org (2012), "Elaborar planes de desarrollo y formar al profesorado local-Xu Lin, directora de Hanban, habla del desarrollo constante del Instituto Confucio”. Disponible en: < http:/spanish.hanban.org/ article/2012-07/31/content_452678.htm> [31 de agosto de 2012].

Struye de Swielande, Tanguy (2009), “La Chine et le 'Soft Power': une manière douce de défendre l’intérêt national?”, núm. 2, Bélgica: Université catholique de Louvain.

Wang, Huning (1993), “Culture as National Soft Power: Soft Power”, en Journal of Fudan University, China.

Wang, Yiwei (2008), "Public Diplomacy and the Rise of Chinese Soft Power”, en Annals of the American Academy of Political and Social Science, vol. 616, Estados Unidos.

Wuthnow, Joel (2008), “The Concept of Soft Power in China’s Strategic Discourse”, en Issues \& Studies, vol. 44, núm. 2, Taiwán: National Chengchi University.

Youming, Wang (2010), “The EU’s Global Governance Versus China’s Harmonious World”, en Real Instituto Elcano de Estudios Internacionales y Estratégicos, núm. 133, Madrid.

Zai China (2010), “El Soft Power de China”. Disponible en: <http:// www.zaichina.net/2010/05/14/el-poder-blando-de-china/> [13 de junio de 2012]. 
Zhongying, Pang (1997), "Soft Power and Others in International Relations”, en Strategy and Management, Núm. 2., China.

Recibido: 29.10.2012

Aceptado: 16.01.2013 